\title{
Editorial for special issue on fuzzy systems in distributed sensing applications
}

\author{
Mohamed Elhoseny ${ }^{\mathrm{a}, *}$ and X. Yuan ${ }^{\mathrm{b}}$ \\ ${ }^{a}$ Faculty of Computers and Information, Mansoura University, Mansoura, Egypt \\ ${ }^{\mathrm{b}}$ Department of Computer Science and Engineering, University of North Texas, USA
}

\begin{abstract}
This special issue of the Journal of Intelligent \& Fuzzy Systems contains selected articles of Fuzzy Systems in Distributed Sensing Applications. It is also containing a collection of interested topics that reflect the latest innovations and research advancements in Innovative Computing, Intelligent Systems, Communication, and Engineering.
\end{abstract}

\section{Introduction}

The interesting features of smart cities have been made possible in recent years because of the availability of commodity low-power sensors, smartphones, tablets, and the necessary wireless networking infrastructure, which, along with technologies such as fuzzy systems, artificial intelligence (AI) and big data processing, might be used to resolve the challenges of sustainable urban environments.

This special issue aims to collect new developments and advances in the area of fuzzy systems in smart sensing applications, and covers the theories, methods, and applications of intelligent communication, as embedded in the fields of engineering and computer science as well as the cross-disciplinary fields.

This special issue contains papers with interesting technical contribution in hot research topics such as:

- Dynamic Analysis and Adaptive Obstacle Avoidance Algorithm of wave Glider Based on Fuzzy Control

- Prediction Method of Cyanobacterial Blooms Spatial-temporal Sequence Based on Deep Belief Network and Fuzzy Expert System

${ }^{*}$ Corresponding author. Mohamed Elhoseny, Faculty of Computers and Information, Mansoura University, Mansoura, Egypt. E-mail: mohamed_elhoseny@mans.edu.eg.
- Predictive Analysis of Impact Hazard Level of Coal Rock Mass Based on Fuzzy Inference Network

- Fuzzy Comprehensive Evaluation of Purchase Intention of Retailer Private Brands Based on Improved AHP method

- A MCCDMA-based Cognitive Radio System for the Internet of Things

- Group Animation Design Technology Based on Artificial Fish Swarm Algorithm

- Artificial Emotion Modeling Based on Container (CUP) Algorithm

- Exploration of Marine Ship Anomaly Real-Time Monitoring System Based on Deep Learning

- Enterprise Patent Management System

All the papers included in this special issue were anonymously reviewed by experts to maintain academic excellence and integrity. We wish to thank all, including authors, reviewers, and all the other participants, who have directly and indirectly contributed to the release of this special issue by their engagement. 